International Mathematical Forum, 2, 2007, no. 9, 413 - 420

\title{
Ranking DMUs with Interval Data Using Interval Super Efficiency Index
}

\author{
F. Hosseinzadeh Lotfi ${ }^{1}$, G. R. Jahanshahloo \\ Dept. of Math., Science and Research Branch, \\ Islamic Azad University, Tehran 14515-775, Iran \\ M. Izadikhah \\ Dept. of Math., Islamic Azad University, Arak, \\ tel:+98-861-3663041-9, P.O. Box 38135/567, Iran
}

\section{Esmaeili}

Dept. of Math., Science and Research Branch, Islamic Azad University, Tehran 14515-775, Iran

\begin{abstract}
In this paper, we present a method for ranking decision making units(DMUs) with interval data in data envelopment analysis. In this method we introduce two efficiency and inefficiency production possibility sets(PPSs),and, using chebychev norm, we measure the distance of each DMU from the frontier of these two PPSs, namely, efficiency and inefficiency frontiers, respectively. Then we rank the DMUs based on their distances from both efficient and inefficient frontiers. Available bank branch data is used to illustrate the applicability of this new approach.
\end{abstract}

\section{Mathematics Subject Classification: 90}

Keywords: Data envelopment analysis, Ranking, Interval data, Efficient frontier, Inefficient frontier

\footnotetext{
${ }^{1}$ Corresponding author: F. Hosseinzadeh Lotfi, E-mail: hosseinzadeh_lotf@@yahoo.com
} 


\section{Introduction}

Data envelopment analysis(DEA) was originally developed to measure the relative efficiency of peer decision making units(DMUs) in multiple input-multiple output settings $[1,2]$. The standard DEA models assume that all data are known exactly without any variation. However, this assumption may not be true.

In the real word, some outputs and inputs may be only known as in forms of interval data, ordinal data and ratio interval data. If we incorporate such imprecise data information in to the standard linear CCR model, the resulting DEA model is a nonlinear and nonconvex program, and is called imprecise DEA(IDEA),(Cooper et al.[3] and Kim et al. [7]). Recently, Despotits and Smirlis [4] calculated upper and lower bounds for the efficiency scores of the DMUs with imprecise data. In Jahanshahloo et al. [5] the radius of stability for the DMUs with interval data is calculated. Also, in Jahanshahloo et al.[6] TOPSIS method for decision-making problems with interval data is extended.

In this paper, we present a new method for ranking decision making units with interval data in data envelopment analysis by introducing two efficiency and inefficiency frontiers and then consider the distances of each DMU from these frontiers in order to rank them. This method is very similar to TOPSIS method, but the rules of the positive ideal and the negative ideal solutions in TOPSIS are played here by the efficiency frontier and the inefficiency frontier, respectively.

The rest of the paper is organized as follows: in section (2) by introducing two production possibility sets and their frontiers we rank all DMUs. In section (3) a numerical example with real interval data are given. A conclusion section summarizes our main results.

\section{Ranking based on efficient and inefficient frontiers}

Suppose we have a set of $n$ DMUs. Each $D M U_{j}(j=1, \ldots, n)$ produces $s$ different outputs $y_{r j}(r=1, \ldots, s)$ utilizing $m$ different inputs $x_{i j}(i=1, \ldots, m)$. Also, assume that input and output levels of each DMUs are not known exactly.

Let $x_{i j} \in\left[x_{i j}^{L}, x_{i j}^{U}\right]$ and $y_{r j} \in\left[y_{r j}^{L}, y_{r j}^{U}\right]$, where lower and upper bounds are known exactly, positive and finite.

First, we introduce two production possibility sets, (PPSs); namely, the effi- 
ciency PPS $\left(T^{U}\right)$ and the inefficiency PPS $\left(T^{L}\right)$ as follows:

$$
\begin{aligned}
& T^{U}=\left\{(X, Y) \mid X \geq \sum_{j=1}^{n} \lambda_{j} X_{j}^{L} \& Y \leq \sum_{j=1}^{n} \lambda_{j} Y_{j}^{U} \& \sum_{j=1}^{n} \lambda_{j}=1 \& \lambda_{j} \geq 0, j=1, \ldots, n\right\} \\
& T^{L}=\left\{(X, Y) \mid X \leq \sum_{j=1}^{n} \lambda_{j} X_{j}^{U} \& Y \geq \sum_{j=1}^{n} \lambda_{j} Y_{j}^{L} \& \sum_{j=1}^{n} \lambda_{j}=1 \& \lambda_{j} \geq 0, j=1, \ldots, n\right\}
\end{aligned}
$$

In Fig. 1 we use one-dimensional input and output data in order to illustrate the efficiency and inefficiency PPSs.

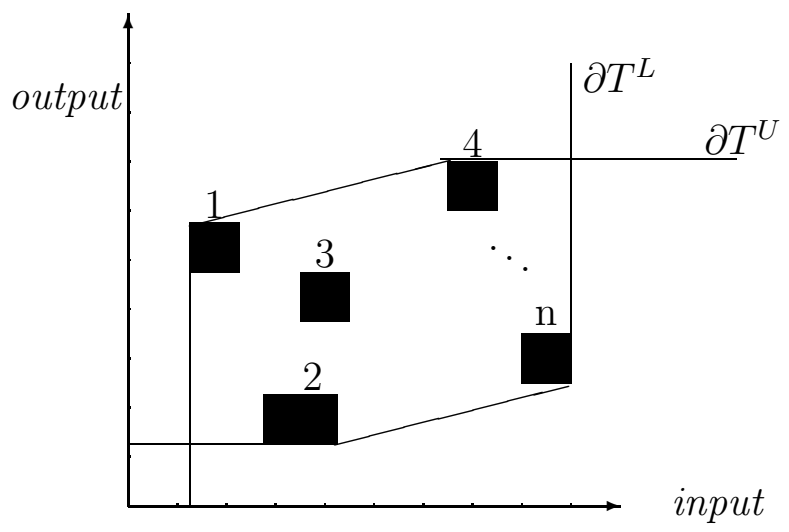

Fig. 1. Efficiency and Inefficiency frontiers

Where $(\partial)$ represents the frontier of production possibility set. Then, we can measure the distance of each $D M U_{t}(t=1, \ldots, n)$ from this two frontiers using the following models:

$$
\begin{aligned}
\max & \alpha_{t} \\
\text { s.t. } & \left(X_{t}^{U}-\mathbf{1} \alpha_{t}, Y_{t}^{L}+\mathbf{1} \alpha_{t}\right) \in T^{U}
\end{aligned}
$$

and

$$
\begin{aligned}
\max & \beta_{t} \\
\text { s.t. } & \left(X_{t}^{L}+\mathbf{1} \beta_{t}, Y_{t}^{U}-\mathbf{1} \beta_{t}\right) \in T^{L}
\end{aligned}
$$

By considering $T^{U}$ and $T^{L}$ we have the two following models:

$$
\begin{aligned}
& \max \alpha_{t} \\
& \text { s.t. } \sum_{j=1}^{n} \lambda_{j} x_{i j}^{L} \leq x_{i t}^{U}-\alpha_{t}, \quad i=1, \ldots, m \\
& \sum_{j=1}^{n} \lambda_{j} y_{r j}^{U} \geq y_{r t}^{L}+\alpha_{t}, \quad r=1, \ldots, s \\
& \sum_{j=1}^{n} \lambda_{j}=1 \\
& \lambda_{j} \geq 0, \quad j=1, \ldots, n
\end{aligned}
$$


and

$$
\begin{aligned}
& \max \beta_{t} \\
& \text { s.t. } \sum_{j=1}^{n} \lambda_{j} x_{i j}^{U} \geq x_{i t}^{L}+\beta_{t}, \quad i=1, \ldots, m \\
& \sum_{j=1}^{n} \lambda_{j} y_{r j}^{L} \leq y_{r t}^{U}-\beta_{t}, \quad r=1, \ldots, s \\
& \sum_{j=1}^{n} \lambda_{j}=1 \\
& \lambda_{j} \geq 0, \quad j=1, \ldots, n
\end{aligned}
$$

Theorem. The models (3) and (4) are feasible and bounded.

Proof:

Clearly, $\left(\lambda_{j}=0, j=1, \ldots, n, j \neq t, \lambda_{t}=1, \alpha_{t}=0\right)$ is a feasible solution for model (3).

By constrains of model (3) we have:

$$
\alpha_{t} \leq \min \left\{-\sum_{j=1}^{n} \lambda_{j} x_{i j}^{L}+x_{i t}^{U}(i=1, \ldots, m), \sum_{j=1}^{n} \lambda_{j} y_{r j}^{U}-y_{r t}^{L}(r=1, \ldots, s)\right\}
$$

and because of $\forall i, r, j \quad x_{i j}^{L}, x_{i j}^{U}, y_{r j}^{L}, y_{r j}^{U}$ are finite, therefore the model (3)is bounded.

Similarly, model (4) is feasible and bounded.

Corollary. Optimal objective values in models (3) and (4) are non-negative. Since $\left(\lambda_{j}=0, j=1, \ldots, n, j \neq t, \lambda_{t}=1, \alpha_{t}=0\right)$ is a feasible solution for model (3) and objective function is in maximization form, thus, $\alpha_{t}^{*} \geq 0$. Similarly, $\beta_{t}^{*} \geq 0$.

In this method, as well as considering the distance of a DMU from the efficiency frontier, its distance from the inefficiency frontier is also considered. That is to say, the less the distance of the DMU under evaluation from the efficiency frontier and the more its distance from the inefficiency frontier, the better its ranking. Suppose $\alpha_{t}^{*}$ and $\beta_{t}^{*}$ are optimal value of objective functions. Therefore, we define the following index, $R_{t}$, for ranking $D M U_{t}, t=1, \ldots, n$

$$
R_{t}=\beta_{t}^{*}-\alpha_{t}^{*}, \quad t=1, \ldots, n .
$$

Therefore, the closer the DMU under evaluation to the efficiency frontier, and farther from the inefficiency frontier, the bigger the value of $R_{t}$.

Therefore, for ranking DMUs using this index, we can rank DMUs in decreasing order. 
It must be noted that, this method for ranking DMUs can easily be used for DMUs with exact data.

Note 1. In the case of exact data, if $D M U_{t}$ be on $\partial T^{U}$, then $\alpha_{t}^{*}=0$ and we have $R_{t}=\beta_{t}^{*}$, and if $D M U_{t}$ be on $\partial T^{L}$, then $\beta_{t}^{*}=0$ and we have $R_{t}=-\alpha_{t}^{*}$. Note 2. Although, this method is very similar to TOPSIS method, but, we don't use the relative closeness measure, $\frac{\beta_{t}^{*}}{\beta_{t}^{*}+\alpha_{t}^{*}}$, that utilizes in TOPSIS method, because for all DMUs on inefficient frontier, in the case of exact data, we have $\beta_{t}^{*}=0$, there for, for these DMUs this relative closeness measure assigns score 0 , thus, we don't able to discriminate between them.

The idea in this method is similar to that in TOPSIS (Technique for OrderPreference by Similarity to Ideal Solution) which is utilized for solving MADM (Multiple Attribute Decision Making) problems.

The difference between TOPSIS and our method lies in the fact that, the rules of the positive ideal and the negative ideal in TOPSIS are played here by the efficiency frontier and the inefficiency frontier, respectively.

\section{Applying this approach to empirical data}

We now apply this new ranking approach to some commercial bank branches in Iran. There are 20 branches in this district. Each branch uses 3 inputs to produce 5 outputs. Table 1 shows the kind of these inputs and outputs.

\begin{tabular}{cc} 
Table 1. & \\
\hline Inputs & Outputs \\
\hline Payable interest & The total sum of four main deposits \\
Personnel & Other deposits \\
Non-Performing loans & Loans granted \\
& Received interest \\
& Fee \\
\hline
\end{tabular}

In Tables 2 and 3 the interval inputs and interval outputs for these DMUs are given. 
Table 2.Input-data for the 20 bank branches

\begin{tabular}{ccccccc}
\hline$D M U_{j}$ & $x_{1 j}^{L}$ & $x_{1 j}^{U}$ & $x_{2 j}^{L}$ & $x_{2 j}^{U}$ & $x_{3 j}^{L}$ & $x_{3 j}^{U}$ \\
\hline 1 & 5007.37 & 9613.37 & 36.29 & 36.86 & 87243 & 87243 \\
2 & 2926.81 & 5961.55 & 18.8 & 2016 & 9945 & 12120 \\
3 & 8732.7 & 17752.5 & 25.74 & 27.17 & 47575 & 50013 \\
4 & 945.93 & 1966.39 & 20.81 & 22.54 & 19292 & 19753 \\
5 & 8487.07 & 17521.66 & 14.16 & 14.8 & 3428 & 3911 \\
6 & 13759.35 & 27359.36 & 19.46 & 19.46 & 13929 & 15657 \\
7 & 587.69 & 1205.47 & 27.29 & 27.48 & 27827 & 29005 \\
8 & 4646.39 & 9559.61 & 24.52 & 25.07 & 9070 & 9983 \\
9 & 1554.29 & 3427.89 & 20.47 & 21.59 & 412036 & 413902 \\
10 & 17528.31 & 36297.54 & 14.84 & 15.05 & 8638 & 10229 \\
11 & 2444.34 & 4955.78 & 20.42 & 20.54 & 500 & 937 \\
12 & 7303.27 & 14178.11 & 22.87 & 23.19 & 16148 & 21353 \\
13 & 9852.15 & 19742.89 & 18.47 & 21.83 & 17163 & 17290 \\
14 & 4540.75 & 9312.24 & 22.83 & 23.96 & 17918 & 17964 \\
15 & 3039.58 & 6304.01 & 39.32 & 39.86 & 51582 & 55136 \\
16 & 6585.81 & 13453.58 & 25.57 & 26.52 & 20975 & 23992 \\
17 & 4209.18 & 8603.79 & 27.59 & 27.95 & 41960 & 43103 \\
18 & 1015.52 & 2037.82 & 13.63 & 13.93 & 18641 & 19354 \\
19 & 5800.38 & 11875.39 & 27.12 & 27.26 & 19500 & 19569 \\
20 & 1445.68 & 2922.15 & 28.96 & 28.96 & 31700 & 32061 \\
\hline
\end{tabular}

Table 3. Output-data for the 20 bank branches

\begin{tabular}{|c|c|c|c|c|c|c|c|c|c|c|}
\hline$D M U_{j}$ & $y_{1 j}^{L}$ & $y_{1 j}^{U}$ & $y_{2 j}^{L}$ & $y_{2 j}^{U}$ & $y_{3 j}^{L}$ & $y_{3 j}^{U}$ & $y_{4 j}^{L}$ & $y_{4 j}^{U}$ & $y_{5 j}^{L}$ & $y_{5 j}^{U}$ \\
\hline 1 & 2696995 & 3126798 & 263643 & 382545 & 1675519 & 1853365 & 108634.76 & 125740.28 & 965.97 & 6957.33 \\
\hline 2 & 340377 & 440355 & 95978 & 117659 & 377309 & 390203 & 32396.65 & 37836.56 & 304.67 & 749.4 \\
\hline 3 & 1027546 & 1061260 & 37911 & 503089 & 1233548 & 1822028 & 96842.33 & 108080.01 & 2285.03 & 3174 \\
\hline 4 & 1145235 & 1213541 & 229646 & 268460 & 468520 & 542101 & 32362.8 & 39273.37 & 207.98 & 510.93 \\
\hline 5 & 390902 & 395241 & 4924 & 12136 & 129751 & 142873 & 12662.71 & 14165.44 & 63.32 & 92.3 \\
\hline 6 & 988115 & 1087392 & 74133 & 111324 & 507502 & 574355 & 53591.3 & 72257.28 & 480.16 & 869.52 \\
\hline 7 & 144906 & 165818 & 180530 & 180617 & 288513 & 323721 & 40507.97 & 45847.48 & 176.58 & 370.81 \\
\hline 8 & 408163 & 416416 & 405396 & 486431 & 1044221 & 1071812 & 56260.09 & 73948.09 & 4654.71 & 5882.53 \\
\hline 9 & 335070 & 410427 & 337971 & 449336 & 1584722 & 1802942 & 176436.81 & 189006.12 & 560.26 & 2506.67 \\
\hline 10 & 700842 & 768593 & 14378 & 15192 & 2290745 & 2573512 & 662725.21 & 791463.08 & 58.89 & 86.86 \\
\hline 11 & 641680 & 696338 & 114183 & 241081 & 1579961 & 2285079 & 17527.58 & 20773.91 & 1070.81 & 2283.08 \\
\hline 12 & 453170 & 481943 & 27196 & 29553 & 245726 & 275717 & 35757.83 & 42790.14 & 375.07 & 559.85 \\
\hline 13 & 553167 & 574989 & 21298 & 23043 & 425886 & 431815 & 45652.24 & 50255.75 & 438.43 & 836.82 \\
\hline 14 & 309670 & 342598 & 20168 & 26172 & 124188 & 126930 & 8143.79 & 11948.04 & 936.62 & 1468.45 \\
\hline 15 & 286149 & 317186 & 149183 & 270708 & 787959 & 810088 & 106798.63 & 111962.3 & 1203.79 & 4335.24 \\
\hline 16 & 321435 & 347848 & 66169 & 80453 & 360880 & 379488 & 89971.47 & 165524.22 & 200.36 & 399.8 \\
\hline 17 & 618105 & 835839 & 244250 & 404579 & 9136507 & 9136507 & 33036.79 & 41826.51 & 2781.24 & 4555.42 \\
\hline 18 & 248125 & 320974 & 3063 & 6330 & 26687 & 29173 & 9525.6 & 10877.78 & 240.04 & 274.7 \\
\hline 19 & 640890 & 679916 & 490508 & 684372 & 2946797 & 3985900 & 66097.16 & 95329.87 & 961.56 & 1914.25 \\
\hline 20 & 119948 & 120208 & 14943 & 17495 & 297674 & 308012 & 21991.53 & 27934.19 & 282.73 & 471.22 \\
\hline
\end{tabular}

Now for ranking these bank branches, first, we consider each of bank branches as a DMU and solve models 3 and 4 for these DMUs. Then by applying the ranking index, $R_{t}, \quad t=1, \ldots, 20$, for each of these DMUs we can rank them. Results are given in Table 4. 


\begin{tabular}{ccccc}
\multicolumn{6}{l}{ Table 4. Results } \\
\hline$D M U_{t}$ & $\alpha_{t}^{*}$ & $\beta_{t}^{*}$ & $R_{t}$ & Rank \\
\hline 1 & 4.4794049 & $1.52906 \mathrm{E}+3$ & 0.9970790 & 5 \\
2 & $1.99789 \mathrm{E}+3$ & $6.07630 \mathrm{E}+2$ & 0.2332084 & 19 \\
3 & 7.2343014 & $1.46836 \mathrm{E}+3$ & 0.9950974 & 7 \\
4 & 2.4257606 & $3.83771 \mathrm{E}+2$ & 0.9937188 & 8 \\
5 & 0.6568042 & 25.9374783 & 0.9753028 & 14 \\
6 & 2.3645638 & $7.11330 \mathrm{E}+2$ & 0.9966869 & 6 \\
7 & 9.2608572 & $1.82759 \mathrm{E}+2$ & 0.9517713 & 17 \\
8 & 2.4191037 & $1.94963 \mathrm{E}+3$ & 0.9987607 & 3 \\
9 & 2.7420434 & 10.3313735 & 0.7902581 & 18 \\
10 & 0.3421236 & 24.8426053 & 0.9864154 & 9 \\
11 & 1.0520472 & $9.61922 \mathrm{E}+2$ & 0.9989075 & 2 \\
12 & 8.8469492 & $3.99007 \mathrm{E}+2$ & 0.9783085 & 12 \\
13 & 7.0673663 & $3.07456 \mathrm{E}+2$ & 0.9775299 & 13 \\
14 & 9.0270694 & $2.39436 \mathrm{E}+2$ & 0.9636683 & 15 \\
15 & 23.3387469 & $1.56537 \mathrm{E}+3$ & 0.9853096 & 10 \\
16 & 11.6377273 & $2.71439 \mathrm{E}+2$ & 0.9588884 & 16 \\
17 & 0.3599989 & $1.57825 \mathrm{E}+3$ & 0.9997720 & 1 \\
18 & 0.3000000 & 14.4128920 & 0.9796097 & 11 \\
19 & 3.8558546 & $1.64013 \mathrm{E}+3$ & 0.9976546 & 4 \\
20 & 15.0950444 & 2.3228109 & 0.1333580 & 20 \\
\hline \multicolumn{5}{r}{}
\end{tabular}

\section{Conclusion}

In this paper, similar with TOPSIS which is utilized for solving MADM problems, first, we introduced two production possibility sets, (PPSs); namely, the efficiency PPS $\left(T^{U}\right)$ and the inefficiency PPS $\left(T^{L}\right)$, and then the distance of each DMU from frontier of these two PPSs measured by chebychev norm. Then we ranked the DMUs based on their distances from both efficient and inefficient frontiers. This ranking method can be used in the case of exact data.

\section{References}

[1] R.D. Banker,A. Charnes,and W.W. Cooper, Some models for estimating technical and scale inefficiencies in DEA, Management Science, 30 (1984) 1078-1092.

[2] A. Charnes, W.W.Cooper, E. Rhodes, Measuring the efficiency of decision making units, European Journal of Operational Research, 2 (1978) 429444.

[3] W.W. Cooper, K.S. Park, G. Yu, IDEA and AR-IDEA: models for dealing with imprecise data in DEA, Management Science, 45 (1999) 597-607.

[4] D.K. Despotis, Y.G. Smirlis, Data envelopment analysis with imprecise data, European Journal of Operational Research, 140(2002)24-36. 
[5] G.R. Jahanshahloo, F. Hosseinzadeh Lotfi, M. Moradi, Sensitivity and stability analysis in DEA with interval data, Applied Mathematics and Computation, 156 (2004) 463-477.

[6] G.R. Jahanshahloo, F. Hosseinzadeh Lotfi, M. Izadikhah, An algorithmic method to extend TOPSIS for decision-making problems with interval data, Applied Mathematics and Computation, 175 (2006) 1375-1384.

[7] S.H. Kim, C.G. Park, K.S. Park, An application of data envelopment analysis in telephone offices evaluation with partial data, Computers and Operations Research, 26(1999) 59-72.

Received: May 1, 2006 